\title{
RECONFIGURACIÓN DE UN ESPACIO SAGRADO: LOS INKAS Y LA PIRÁMIDE PUMAPUNKU EN TIWANAKU, BOLIVIA
}

\author{
RECONFIGURING SACRED SPACE: THE INKAS AND \\ THE PUMAPUNKU PYRAMID AT TIWANAKU, BOLIVIA
}

\author{
Jason Yaeger* y José María López Bejarano**
}

\begin{abstract}
Las crónicas dan cuenta que Tiwanaku fue una sede de gran importancia política para el imperio Inka, pues en ésta residía un gobernador imperial, sиyoyoc apu; de igual forma hacen mención a la existencia de un palacio real, en el cual presumiblemente nació Manco Capac, hijo de Huayna Capac. Los Inkas consideraron a Tiwanaku un sitio sagrado de gran relevancia, a la par con la Isla del Sol y Pachacamac. Según uno de los relatos Inkas referidos a la Creación, Tiwanaku fue el sitio en el que Viracocha dio origen a las parejas primigenias de todas las etnias. Los edificios y esculturas existentes en el sitio constituían pruebas concretas de estos sucesos, y es en este marco que proponemos discutir el rol de Tiwanaku en la ideología Inka. El presente artículo se enfoca en la pirámide de Pumapunku, con el objetivo de entender la razón que impulsó a los Inkas en la elección de esta estructura como el núcleo ritual de su asentamiento en la región, y demostrar a la vez cómo modificaron dicho complejo de acuerdo a su cosmología y su historia mítica. Se propone, además, un marco interpretativo para ayudar a comprender cómo la materialización y manifestación de dicha historia mítica, sumada a las actividades efectuadas en el espacio reconfigurado, ayudaban a legitimizar el dominio ejercido por los Inkas sobre las poblaciones andinas.
\end{abstract}

Palabras claves: Inka, Tiwanaku, Pumapunku, ideología, sitio ceremonial, imperialismo.

Spanish chronicles tell us that Tiwanaku was an important political center for the Inka Empire. One of the two imperial governors (suyoyoc apu) lived there, and the site contained an Inka royal palace, where Huayna Capac planned the suppression of the Quito rebellion and where his son, Manco Capac, was born. The sacred character of Tiwanaku complemented and contributed to the site's political importance. One Inka creation narrative holds that Viracocha created the primordial couples for all Andean ethnic groups at Tiwanaku before sending them out to populate their respective homelands. The abandoned Middle Horizon buildings and sculptures constituted physical proof of these creative acts, which made Tiwanaku a sacred center on a par with the Island of the Sun and Pachacamac. In this paper, we discuss the role of Tiwanaku in Inka imperial ideology, focusing on the Pumapunku pyramid and associated structures. We discuss the reasons that the Inkas chose the Pumapunku over other monumental structures to be the ritual focus of their settlement, and then examine the ways in which they modified the Pumapunku to correspond with their cosmology, their mythic history, and the specific requirements of Inka ritual practice. We conclude with a discussion of the ways in which the materialization of Inka cosmology and history at Tiwanaku through architecture and ritual practice was one tool in Inka imperial strategies to legitimate the extension of Inka political sovereignty over other Andean peoples.

Key words: Inka, Tiwanaku, Pumapunku, ideology, ceremonial center, imperialism.

En todo el mundo, los lugares sagrados tienden a conservar su esencia sagrada a pesar del transcurso del tiempo; este fenómeno es palpable particularmente en sitios que ostentan una notable presencia física y una apariencia que los hacen sobresalir del paisaje (Bradley 1998). El contenido cultural de los sitios sagrados, es decir el significado que la gente atribuye a éstos y/o las interpretaciones con las que son entendidos, no se mantienen fijos y estables, por el contrario pueden transformarse de una manera muy significativa con el paso de los siglos (Bradley 2000). Un lugar sagrado, ya sea éste un rasgo "natural" del paisaje o una estructura, conlleva a algunos significados provocados por la observación e interpretación de sus características físicas, en este caso nos referimos a su forma, tamaño, volumen, color, textura, etc. A la vez, las actividades que ejecutan las personas en estos lugares aportan también otros significados, y la interpretación del lugar se va transformando por una dinámica dialéctica entre los modos en que la gente entiende al lugar y las experiencias vividas

\footnotetext{
* Facultad de Antropología, Universidad de Wisconsin, 5240 Social Science Building, Madison, WI 53706-1393, EE.UU.

** Dirección Nacional de Arqueología, DINAR, Calle Tiwanaku 93, La Paz, Bolivia.
} 
en dicho sitio. Es por medio de esta dialéctica que un lugar llega a adquirir fuerza social (Alcock 2001; Basso 1996; Bradley 1998).

De igual forma, el significado de un lugar sagrado puede sufrir modificaciones cuando es interpretado o reinterpretado por una nueva cultura, la cual trae consigo una cosmología e ideología propias. En la larga historia precolombina de Sudamérica muchas sociedades incorporaron los sitios sagrados de sociedades precedentes a su mundo mítico-religioso, y entre ellas, ninguna lo hizo con más energía y eficacia que los Inkas, para quienes la incorporación de lugares sagrados y objetos ceremoniales servía como una estrategia de expansión imperial. En algunos sitios, como Pachacamac (Rostworowski de Diez Canseco 1992), los Inkas modificaron o añadieron a los espacios sagrados preexistentes características propias de su cultura. En otros, como la Isla del Sol y Catequil, reemplazaron los edificios y espacios sagrados precedentes, reconfigurando totalmente la organización del espacio cúltico (Bauer y Stanish 2001; Topic et al. 2002). En este marco, el presente artículo propone discutir el modo en el que los Inkas interpretaron y reorganizaron el espacio sagrado en Tiwanaku. A continuación del resumen del programa de investigaciones del asentamiento Inka en Tiwanaku, esbozamos una serie de explicaciones que permiten entender las razones que provocaron la elección de la pirámide de Pumapunku como foco central y ritual del asentamiento Inka. Posteriormente enfatizamos las modificaciones hechas en dicho complejo, las cuales entendemos responden a la cosmología, historia mítica, y las necesidades rituales Inkas. Subsecuentemente, concluimos con una discusión centrada en explicar cómo la materialización de dicha cosmología e historia mítica, palpable y visible en la arquitectura, combinada con las manifestaciones y las actividades efectuadas en el espacio reconfigurado, contribuyeron a la legitimización del dominio ejercido por los Inkas sobre los pueblos andinos.

\section{La Urbe de Tiwanaku en el Incario}

Ubicado en el altiplano, a 3.850 metros sobre el nivel de mar, Tiwanaku llegó a dominar un área de los Andes que se extendía desde el altiplano boliviano hasta la costa peruana (Figura 1). Gracias a las investigaciones realizadas por diversos arqueólogos -entre ellos Carlos Ponce Sanginés y
Alan Kolata- durante dos siglos sabemos mucho sobre el desarrollo de Tiwanaku como un gran centro urbano (e.g., Albarracín-Jordan 1996, 1999; Escalante 1997; Janusek 1994, 1999; Kolata 1993, 1996, 2003; Kolata y Ponce 1992; Ponce 1972, 1999; Vranich 1999). Durante su apogeo en el Horizonte Medio, Tiwanaku fue la ciudad más grande de Sudamérica, y unos 30.000 habitantes vivían alrededor del núcleo urbano compuesto por templos, plazas públicas y palacios reales. Esta urbe tuvo a la pirámide Akapana (Figura 2), la cual mide casi 17 metros de alto, como una de sus máximas representaciones monumentales (Kolata 1993; Manzanilla 1992; Vranich 2001). Al norte de ella se ubican Kalasasaya y el Templete Semisubterráneo, estructuras que forman el corazón de Tiwanaku (Ponce 1981). A un kilómetro del área central se halla la estructura de Pumapunku, la cual muestra como base una plataforma en T, que sobrepasa en extensión a la pirámide de Akapana, llegando a medir 150 metros de lado (Escalante 1997; Kolata 1993; Vranich 1999). Esta zona nuclear de estructuras monumentales se encontraba rodeada por unidades residenciales construidas con materiales más perecederos y constituían a la vez la mayoría de las construcciones de la ciudad (Janusek 1994, 2004; Kolata 1992, 2003; Ponce 1972; Parsons 1968).

Las construcciones monumentales, anteriormente citadas, reflejan el poder y la influencia panregional ejercida por Tiwanaku durante el Horizonte Medio (Berenguer y Dauelsberg 1989; Browman 1981, 1997; Cook 1994; Kolata 1993; Stanish 2002), pero hacia el 1.150 d.C., el centro cívicoceremonial yacía prácticamente abandonado por causas todavía desconocidas (véase la discusión de Brennan et al. 1997; Erickson 1999; Ortloff y Kolata 1993). Sin importar las razones por las cuales se abandonó la ciudad, gran parte de la urbe se hallaba en ruinas cuando el Imperio Inka sometió la zona al sur del lago Titicaca hacia el 1.470 d.C. Sobre la condición de la ciudad, cabe realizar tres observaciones básicas pero importantes:

Primera. Las grandes plataformas cuidadosamente construidas seguramente continuaban en pie a la llegada de los Inkas, sobreviviendo siglos, para luego ser descritas por los cronistas españoles en los siglos XVI y XVII (resumidos en Kolata 1993). Probablemente habrían sufrido el colapso y desintegración los complejos residenciales construidos con muros de tapial y adobe donde residía la mayo- 


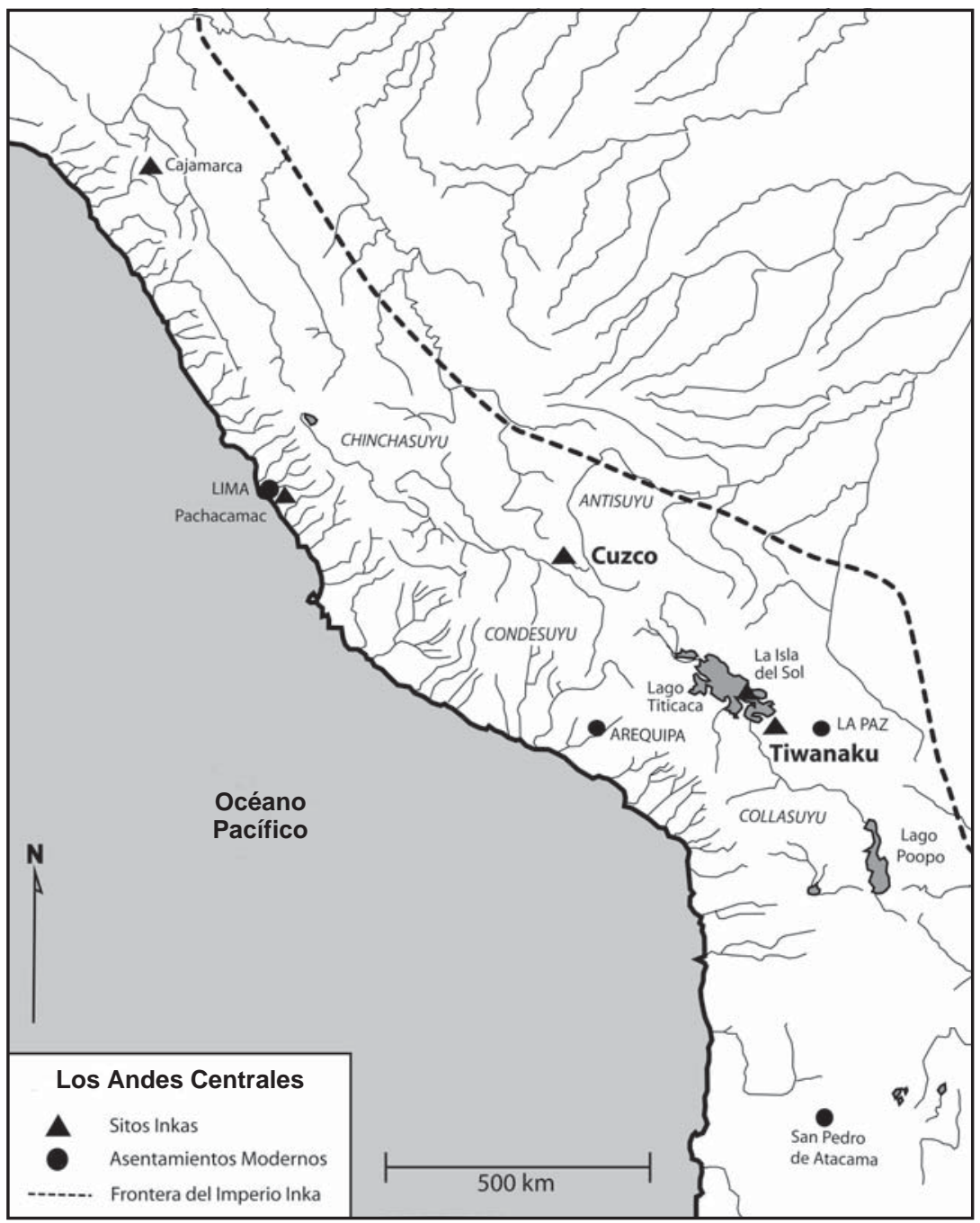

Figura 1. Los Andes Centrales, mostrando la parte central del imperio Inka. The Central Andes, showing the central portion of the Inka empire.

ría de la población (véase referencias anteriormente citadas). En cambio, la arquitectura monumental habría sufrido menos el efecto degradante del tiempo, tanto por los materiales con que estaba construida como por las técnicas usadas en su confección.

Segunda. A pesar de conservar su forma original, Pumapunku había sufrido bastantes modificaciones antes de la llegada de los Inkas. El proceso de desmantelamiento de Pumapunku comenzó en la época Tiwanaku V o en el período Intermedio Tardío, cuando unas ofrendas dedicatorias dispuestas por debajo de los cimientos de la plataforma fueron saqueadas (Vranich et al. 2001). Al mismo tiempo, los muros de retención en la plataforma se desmantelaron, hecho observable por la construcción de estructuras Inkas en áreas que habían sostenido muros de construcción Tiwanaku durante el Horizonte Medio (Vranich et al. 2001). Este fenómeno no se restringió a $P u$ mapunku, y estudios cuidadosos del resto de las plataformas monumentales en Tiwanaku han demostrado que algunas de éstas fueron construidas con bloques tallados removidos de otras plataformas preexistentes, que fueron parcialmente desmanteladas durante el Horizonte Medio (Vranich comunicación personal 2004).

Tercera. En el sitio se observaban aún muchas piezas escultóricas en pie o desmoronadas. El área 
de Pumapunku mostraba aún importantes piezas con frisos arquitectónicos de impresionante factura, ejemplos claros son los diseños que presentan la cruz andina, asociados a grandes portales similares a la Puerta del Sol (Protzen y Nair 2001, 2002; Vranich 1999). Por otra parte, reportes tempranos en el sitio hacen referencia a la presencia de un monolito próximo a la plataforma de Pumapunku, el cual se hallaba en pie al oeste de los grandes bloques de piedra que componen el frontis de la estructura (Grant 1893:4; Vranich 1999).

Con estos referentes, es necesario hacer notar que, si bien el grado de destrucción presente en la urbe Tiwanakota era relevante durante el Intermedio Tardío, muchas de las estructuras aún en pie conservaban características apreciables a la llegada de los Inkas. Esto, como es de esperarse, no implicó una reducción en la veneración sentida por los Inkas, ni involucró un cambio sustancial en la concepción que tenían éstos del sitio como un centro sagrado. De hecho, éste se hallaba a la par con la Isla del Sol y Pachacamac. Es más, una narración Inka que describe la Creación postula que fue en Tiwanaku donde el creador Viracocha trajo al mundo a las parejas ancestrales de todos los grupos étnicos, y que de ahí los envió a poblar la tierra (Betanzos 1987 [1551-1557]; Sarmiento de Gamboa 1967 [1572]). Juan de Betanzos (1987 [15511557]), cronista del siglo XVI, nos relata que para los Inkas, los restos de la urbe de Tiwanaku representaban la evidencia palpable de esta Creación. En particular, sostiene que los Inkas creían que las esculturas presentes en el sitio no eran otra cosa que arquetipos usados por Viracocha en la creación de las parejas originarias.

De esta manera, tanto las esculturas como los edificios constituían la prueba física que sustentaba la creencia que Tiwanaku era el sitio de la Creación de las parejas originarias y las etnias andinas. De esta forma el Imperio Inka al mantener bajo su dominio la cuna sagrada de origen y el centro del orden simbólico pretendía imponer control sobre los descendientes de esas parejas primordiales.

No debe sorprender bajo estas circunstancias que Tiwanaku hubiese llegado a ser considerado un centro político de suma importancia durante el periodo Inka, en virtud al importante nexo que este sitio tenía con la historia de los Inkas y los pueblos sujetos al imperio. Tan importante fue, que los Inkas consideraron construir su capital ahí y diseñaron Cuzco bajo los parámetros arquitectónicos obser- vados en Tiwanaku (e.g., Cieza de León 1959 [1553]). Y a pesar de no haber llegado a erigirse como la capital, Tiwanaku quedó como una huaca importante para el imperio (Albornoz 1989 [15811585]). Al respecto, Cieza de León (1959 [1553]) relata que Tiwanaku fue una sede de gran importancia para el ejercicio del poder político Inka, ya que en esta residía uno de los dos Suyoyoc Apu, Gobernadores Generales del Imperio. Sostiene también que en el sitio se alzaba un palacio real donde el emperador Huayna Capac formuló su campaña para aplastar a las fuerzas sublevadas en Quito y fue también en Tiwanaku donde nació su hijo Manco Inka (Cieza de León 1959 [1553]). En cuanto a la cultura material, otros autores han hecho hincapié en la tendencia Inka de apropiar formas tiwanakotas no solamente en la mampostería de las estructuras, sino también en la reproducción de formas cerámicas como el kero (Browman 1978; Cummins 2002).

\section{Investigaciones del Asentamiento Inka en Tiwanaku}

Tiwanaku nos brinda la oportunidad de estudiar el rol de la historia mítica y la cosmología inmersas en la empresa imperial de los Inkas, a través del estudio de los restos físicos existentes en la actualidad. Hasta la fecha, son numerosos los investigadores que han hallado restos arqueológicos de filiación Inka en Tiwanaku, especialmente en el área de Pumapunku, entre ellos Gregorio Cordero Miranda en 1977 y 1978, e investigaciones más amplias de la DINAR (Dirección Nacional de Arqueología de Bolivia) en 1989 (Arellano 1991; Cordero 1978; Escalante 1997). Los trabajos de la DINAR lograron descubrir numerosos rasgos arquitectónicos con clara asociación a cerámica de filiación Inka y colonial. Entre los edificios, se destaca una estructura rectangular próxima a la fachada norte de Pumapunku, también una estructura cuadrangular semejante a un estanque en el lado occidental de Pumapunku y recintos en la primera terraza de la estructura hacia el noreste.

Sin embargo, quedó sin explorar de manera específica la ocupación Inka y colonial de Tiwanaku hasta que dimos inicio al programa de investigaciones del asentamiento Inka en 1999, que forma parte del Proyecto Arqueológico Pumapunku-Akapana encabezado por el doctor Alexei Vranich.

A fin de aproximar la investigación a las cuestionantes planteadas por el proyecto, nuestro tra- 
bajo propuso tres objetivos principales: 1) determinar el tamaño y la organización del asentamiento Inka; 2) definir el estado en que se encontraba Pumapunku a la llegada de los Inkas y las remodelaciones que ellos realizaron en la estructura monumental; y 3 ) identificar la función de las estructuras que los Inkas erigieron en el área de Pumapunku.

De la misma manera, el proyecto propuso tres componentes de investigación más específicos: 1) prospección de superficie del asentamiento Inka; 2) prospección geofísica del área alrededor de $P u$ mapunku; 3 ) trabajos de excavación extensiva y en área, en el asentamiento ubicado al norte de $P u$ mapunku (Figuras 2 y 3 ).

A través de cuatro temporadas de trabajo, entre 1999 y 2002, se logró cumplir las metas planteadas, y la investigación ha proporcionado muchos datos nuevos y relevantes con respecto al asentamiento Inka en Tiwanaku (Vranich et al. 1999; 2001). Con este referente, podemos hacer algunas observaciones puntuales.

\section{El Papel Central de Pumapunku en el Asentamiento Inka}

Nuestras investigaciones permiten la reconstrucción del tamaño y organización del asentamiento Inka en Tiwanaku. Información obtenida gracias a la prospección de superficie llevada a cabo por Smith (2002) indica que el asentamiento Inka abarca un área que cubre alrededor de $1,7 \mathrm{~km}^{2}$ (Figura 2). Gracias a colecciones sistemáticas, Smith identificó la existencia de tres zonas distintas de ocupación durante el Horizonte Tardío. La más grande es una zona caracterizada por la alta densidad de tiestos, en superficie, de estilo imperial, incluyendo una gran cantidad de platos y aríbalos, que se extiende desde Pumapunku hasta las proximidades del pueblo actual y creemos constituye el centro político y ritual Inka. $\mathrm{Al}$ noreste se ubica otra zona que presenta una alta densidad de fragmentos correspondientes a tinajas, aríbalos, y otras vasijas para almacenamiento, lo cual puede representar la existencia de un tambo u otra instalación imperial, ésta, por cierto, se

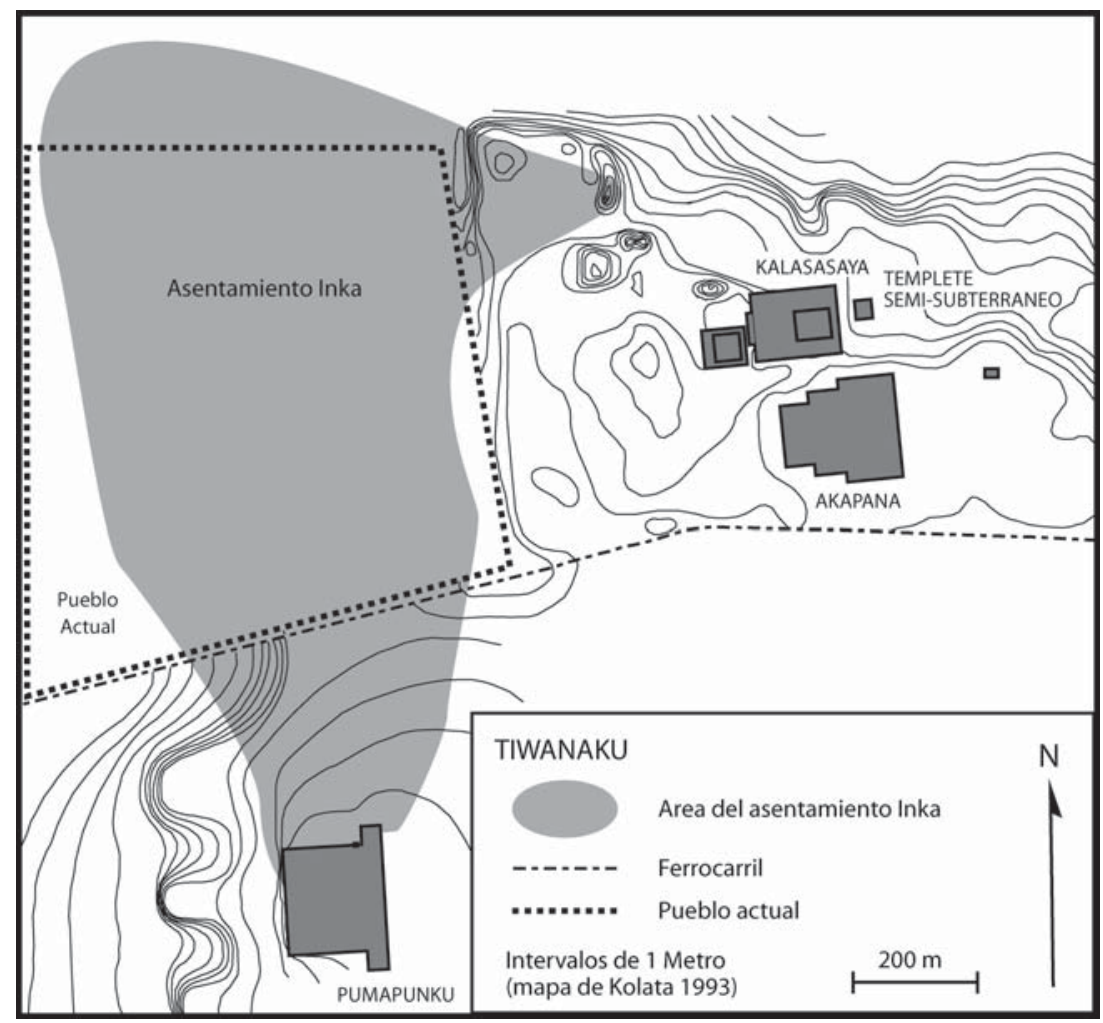

Figura 2. Tiwanaku (basado en Kolata 1993). Map of Tiwanaku (after Kolata 1993). 


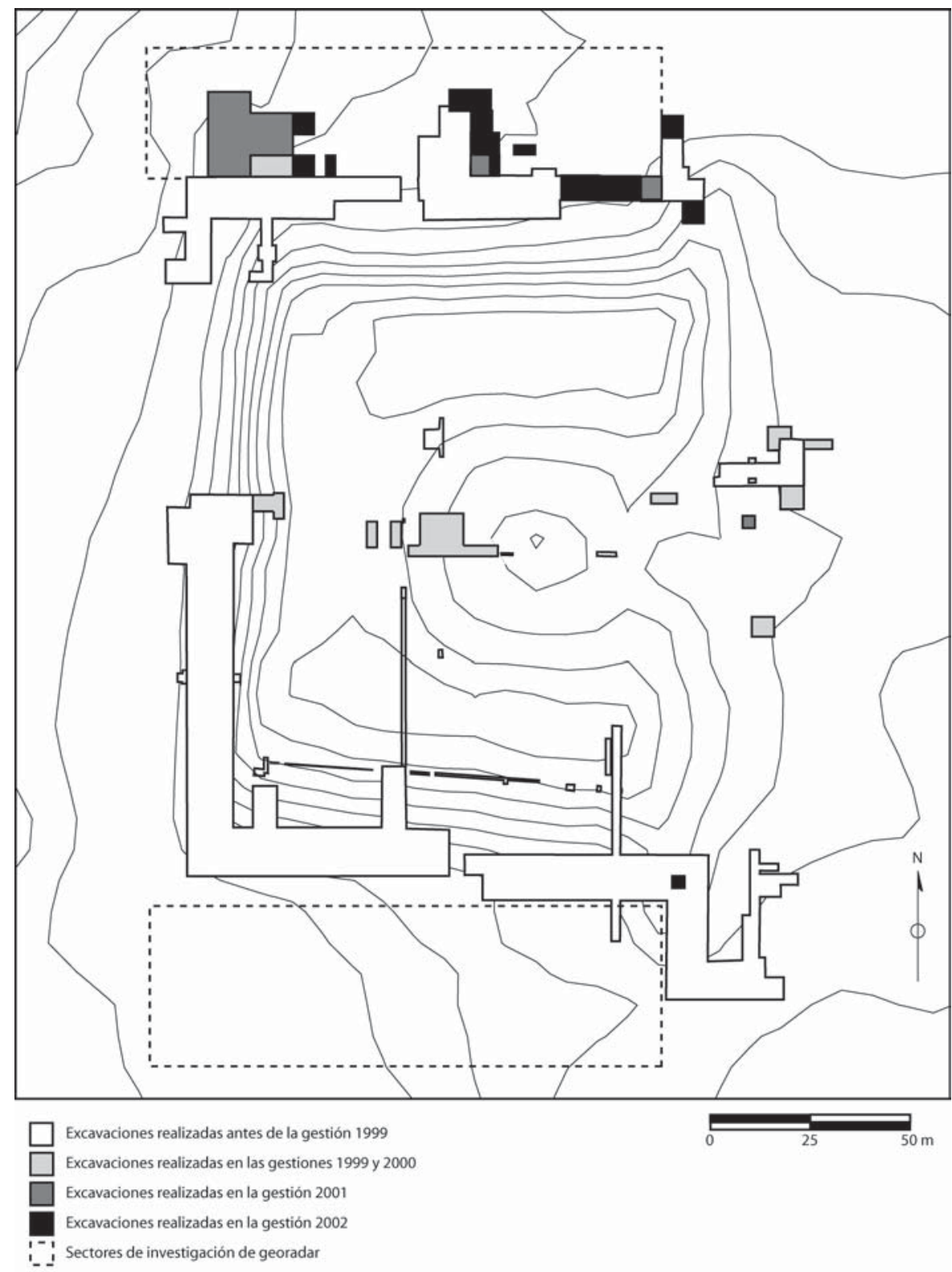

Figura 3. Excavaciones en la pirámide de Pumapunku Excavations of the Pumapunku

halla adyacente a la vía imperial Inka. Finalmente se observó la existencia de una zona residencial donde se asentaba la población indígena local, la cual se caracteriza por una frecuencia alta de cerámica Pacajes (Smith 2002).

Los resultados obtenidos por Smith confirmaron nuestra hipótesis, que suponía que los Inkas no ocuparon de manera significativa la zona monumental de la urbe de Tiwanaku, por el contrario, de todos los edificios monumentales existentes a su llegada, los Inkas se interesaron en una sola estructura grande, Pumapunku, hecho que también es mencionado por Cobo (1939 [1653]: 41-42, traducido en inglés en Cobo 1990: 105):

...tuvieron por templo célebre el sobredicho de Pumapuncu, y lo ilustraron y enriquecieron, acrecentando su ornato y el 
número de ministros y sacrificios; y edificaron junto a él palacios Reales en que dicen nació Manco-Capac, hijo de Guayna-Capac, cuyas ruinas se ven hoy; y era edificio muy grande y de muchas piezas y apartamentos.

La carencia de arquitectura y materiales de filiación Inka alrededor de Akapana y Kalasasaya propone la necesidad de explicar el porqué los Inkas decidieron asentarse exclusivamente en Pumapunku y no en el área central de la urbe. Tanto Akapana como Kalasasaya debieron presentar fachadas y acabados impresionantes en el Intermedio Tardío y es muy probable que Pumapunku no hubiese sido la estructura más portentosa de Tiwanaku durante este período. Este hecho nos hace cuestionar ¿cuál fue la razón que indujo a los Inkas para la elección de Pumapunku como el centro de su asentamiento? Al respecto proponemos cinco posibles motivos.

Primero. Vranich (1999) ha observado que el eje de Pumapunku se alinea de forma directa con el pico sagrado Illimani. Esta alineación es observable en la actualidad, y dado el afán Inka por construir edificios que imitaban puntos importantes en el paisaje, nos parece muy probable que los Inkas hubiesen notado este fenómeno extraordinario, afectando su decisión de incorporar Pumapunku en su asentamiento.

Segundo. El mismo eje central de Pumapunku se alinea con el pico Illimani, y relaciona el este, al amanecer y el mundo celestial con el Lago Titicaca, de la misma manera, asocia el oeste al atardecer y al inframundo. Kolata (1993) ha sugerido que la alineación este-oeste entre el pico y el lago reproducía el movimiento del sol y a la vez unía los varios pares de conceptos dualistas asociados con los dos extremos del camino del sol y las divisiones aymaras de uma y urco. Gracias a esta ubicación, Pumapunku adquiere importancia como un axis mundi que conectaba los varios mundos del cosmos (Vranich 1999).

La forma de la estructura en sí también sugiere su rol como un axis mundi. En este caso hacemos referencia al alajpacha, mundo celestial, representado por la pirámide misma, el manqapacha, inframundo de contacto con los seres subterráneos, representado por el patio hundido ubicado en su cima, y el mankapacha, mundo terrenal, en el que se desenvuelven los seres huma- nos, representado por la plaza ubicada hacia el este. Un concepto similar ha sido ya expuesto por Burger (1992) para contextos más tempranos, y Zuidema (1990) propone que el Inka ushnu se entendía de la misma manera. En este aspecto, vale señalar que Vranich y Yaeger (2003) han postulado que Pumapunku tiene una relación íntima con la concepción del ushnu Inka. Proponen de igual manera que la forma de la estructura, semejante a las puertas emblemáticas de Tiwanaku (Vranich 1999), se asocia con el concepto de ush$n u$ como portal y nexo entre los mundos antes descritos.

Tercero. Moore (1996) ha demostrado que las ceremonias Inkas requerían espacios extensivos, y por tal razón requerían de la construcción de plazas mucho más grandes que aquellas observadas en civilizaciones anteriores. La única plaza grande conocida en Tiwanaku se ubica inmediatamente al este de Pumapunku. Mide $150 \mathrm{~m}$ por lado, y gracias a su tamaño, bien pudo ser usada en las ceremonias Inkas.

Cuarto. El patio hundido central de Pumapunku era un receptáculo natural de agua. Por tal motivo los tiwanakotas construyeron canales de desagüe, los cuales sufrieron modificaciones con el transcurso del tiempo. A su llegada los Inkas crearon también un sistema de canalización para dirigir el líquido a un tanque y luego a un baño ritual, que habría de tener uso restringido y sagrado, como se ha observado en otros casos similares de ritos Inkas (Sherbondy 1992). Al respecto, sugerimos que el manejo de aguas a través del sistema de canales también puede demostrar una compleja concepción de los espacios sagrados, toda vez que los ríos y canales representan también al mundo subterráneo de acuerdo a la cosmología aymara.

Quinto. El monolito asociado a la cara oeste de Pumapunku (Grant 1893:4), ya removido, con certeza encajó muy bien con la creencia Inka que suponía que las esculturas antropomorfas eran modelos para las parejas originales creadas en Tiwanaku por Viracocha como nos cuenta Betanzos (1987 [1551-1557]).

Por estos cinco hechos físicos en Pumapunku, que abarcan su morfología arquitectónica, su ubicación dentro del plan urbano de Tiwanaku y su relación con el paisaje sagrado de la región, suponemos que se presentó para los Inkas como el mejor candidato en la concepción de sitio ritual y sagrado. Sin embargo, y a pesar de este hecho, 
los Inkas tuvieron que modificar la estructura y perfeccionarla para que se ajustara con sus necesidades rituales, debido a que originalmente la pirámide había sido diseñada de acuerdo a los requisitos espaciales de los rituales de Tiwanaku y había sufrido daños por el saqueo y el deterioro natural, ocurridos con anterioridad a su llegada. Por tal razón, depositaron una línea de ofrendas dedicatorias en el lado este de la pirámide, y éstas incluían en la mayoría de los casos tejidos, vasijas miniaturas y objetos de cobre asociados a restos humanos pertenecientes a infantes. Tomándose como caso particular el de una ofrenda ubicada en el eje central de la plaza, en la que se destaca el hallazgo de los restos óseos de un adolescente (Figura 4). Con estas ofrendas y el baño ya mencionado, los Inkas reedificaron y reconfiguraron a Pumapunku como un lugar sagrado.

\section{Actividades Inkas asociadas con Pumapunku}

El asentamiento Inka tiene como núcleo el sector norte de Pumapunku, como se aprecia en la Figura 2, y constituyó el centro de nuestras excavaciones (Figura 3). Estas investigaciones revelaron una diversidad sorprendente de áreas de actividad alrededor de Pumapunku, siendo su identificación el resultado de la excavación sistemática de aproximadamente 750 metros cuadrados, a lo que se suma el análisis de los artefactos procedentes de la zona. De esta forma, identificamos cinco tipos distintivos de espacios. La organización de la arquitectura y nuestras observaciones preliminares nos indican que existen zonas de actividades distintas, las cuales describimos a continuación:

La primera zona es una sala formal, tal vez una residencia o quizás una sala de audiencias, de aproximadamente 6 × 5 m (Figura 5). La misma, presenta semejanzas con el resto de las estructuras en el asentamiento Inka, es decir, los muros muestran un cimiento compuesto por piedras dispuestas a doble hilera, los muros hechos de abobe y tapial se ubicaban encima. Sin embargo, a diferencia de las demás estructuras, ésta se distingue por sus características internas. Presenta un piso preparado de arcilla con áreas elevadas, entre ellas, bancos o banquetas adosadas a los muros sur y norte y pedestales elevados de arcilla en las cuatro esquinas. $\mathrm{Al}$ norte y noreste de la sala formal y conectadas a ella por pasillos angostos se ubican por lo menos

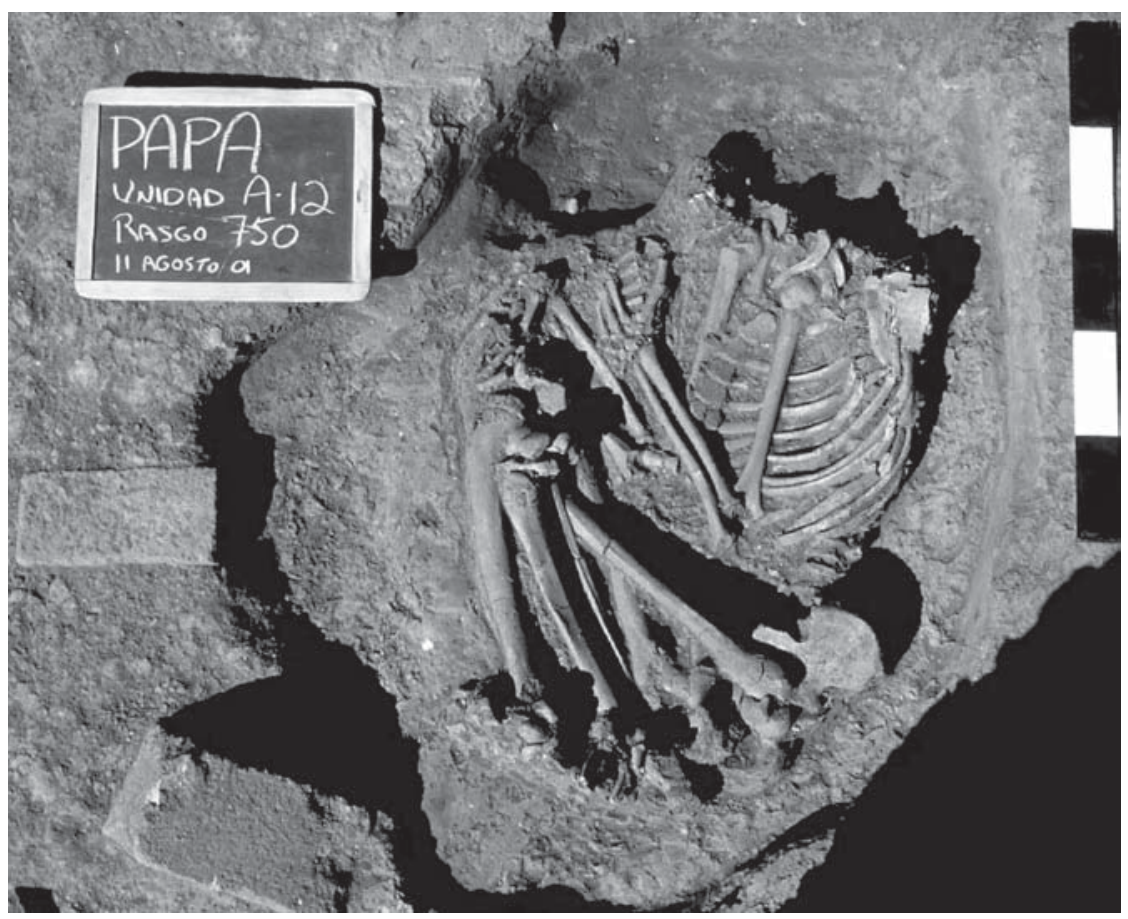

Figura 4. Ofrenda Inka (Rasgo 750) en el lado oriental de Pumapunku. Inka offering (Feature 750) on the east side of the Pumapunku. 


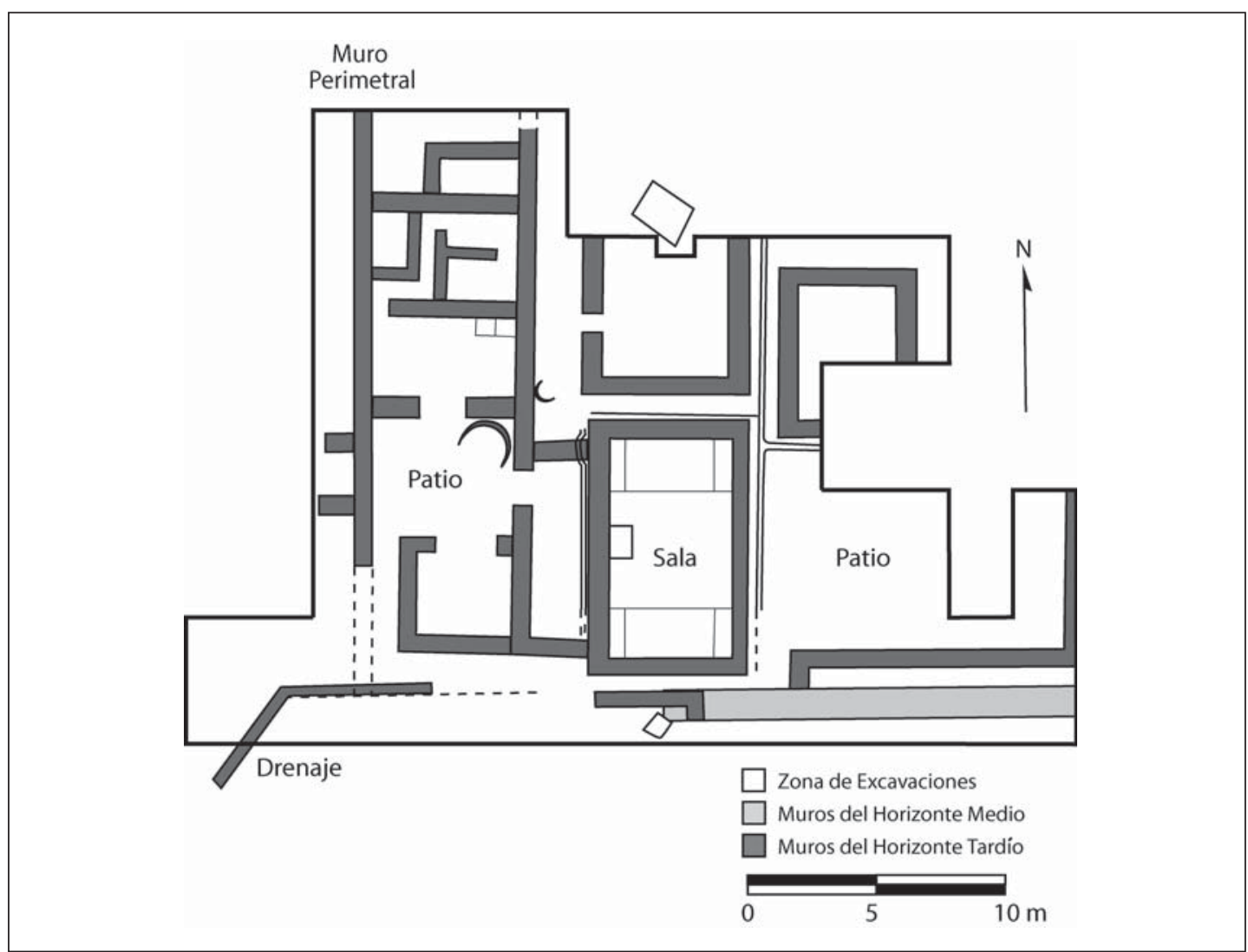

Figura 5. Excavaciones en la zona noroeste de Pumapunku. Excavations in the northwest zone of the Pumapunku.

dos estructuras más cuadrangulares de tamaño semejante a la sala pero sin el acabado fino. Al este de la sala se aprecia un patio abierto que excede en tamaño a los patios hallados hacia el oeste.

A la vez, un pasaje corre desde la esquina suroeste de dicho patio y pasa entre la sala y la primera terraza de Pumapunku hasta llegar a la segunda área de actividad, destinada a la preparación y almacenaje de comida. En ésta, tres cuartos de área reducida, aproximadamente $2 \times 3 \mathrm{~m}$, rodean un patio empedrado de dimensiones modestas (Figura 5). En el rincón noreste de este se encuentra un rasgo llamativo, en forma de fuente, que presenta un diámetro aproximado a $2 \mathrm{~m}$, con dos capas delgadas de carbón. Dicho rasgo está asociado a su vez a una capa de huesos de camélidos, pescados y roedores, en su mayoría quemados; a este contexto se suman algunos tiestos de aríbalos y cerámica doméstica, distribuidos en la superficie del patio, lo cual sugiere que esta área fue utilizada para la preparación de alimentos. Por otra parte, existen en el extremo norte de este sector algunas pequeñas áreas techadas, aproximadamente 1 x $1 \mathrm{~m}$, usadas posiblemente para el almacenaje de víveres.

Un muro más amplio que los de las estructuras comunes cierra esta zona en su lado occidental, probablemente rodeando la zona entera y sirviendo de perímetro.

Aproximadamente $20 \mathrm{~m}$ al este de la sala formal se ubica la tercera zona, la cual fue parcialmente excavada por Javier Escalante M. y Leocadio Ticlla en 1989. En ésta se emplazan cuatro edificios grandes, de forma rectangular, de aproximadamente 4 x 10 m (Figura 6). En dos de éstos se hallaron impresionantes depósitos de aríbalos y ollas para cocinar, asociados a restos óseos de camélidos, aves, pescados, y abundante carbón (Figura 7). Al mismo tiempo, en los corredores que articulan los cuatro edificios se hallaron depósitos semejantes. Los contenidos en estos depósitos parecen provenir de la preparación de alimentos, usa- 


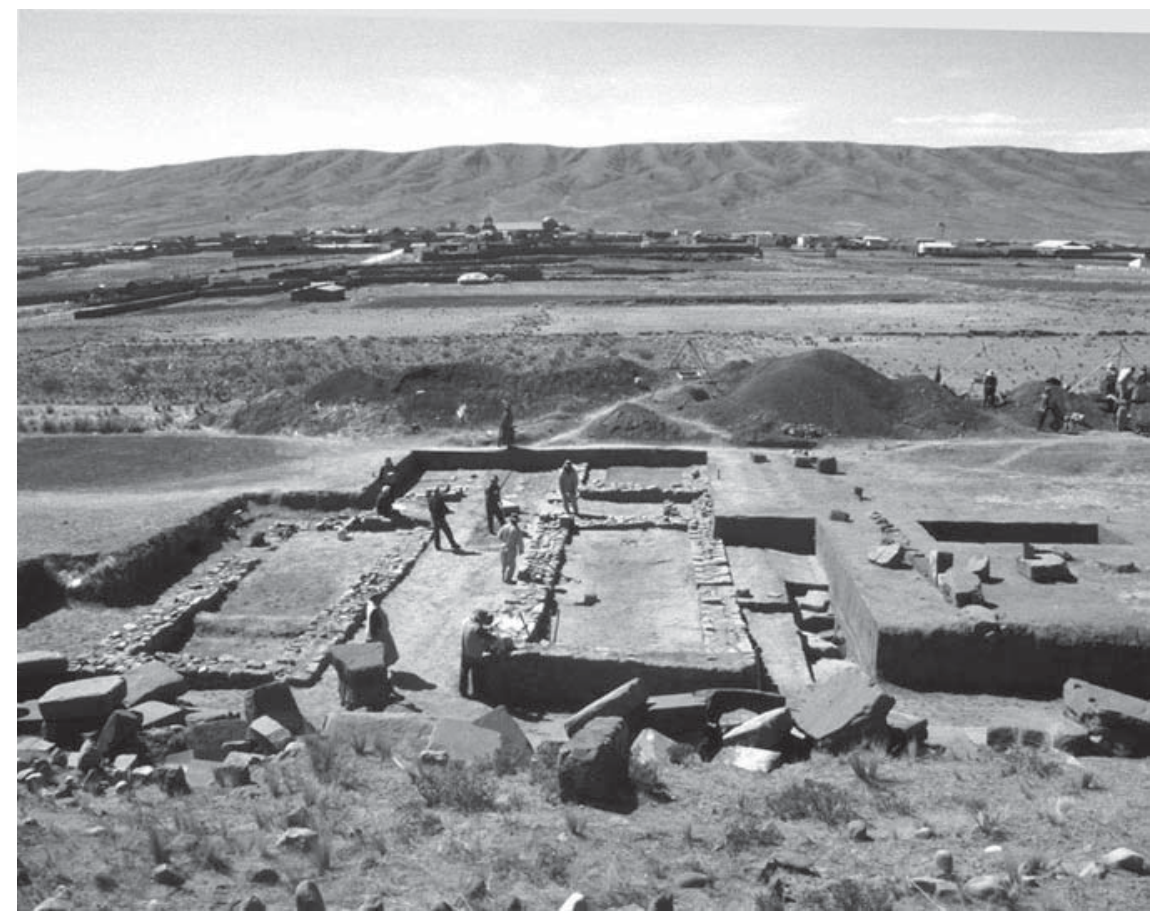

Figura 6: Excavaciones en la zona norcentral de Pumapunku. Excavations in the north-central zone of the Pumapunku.

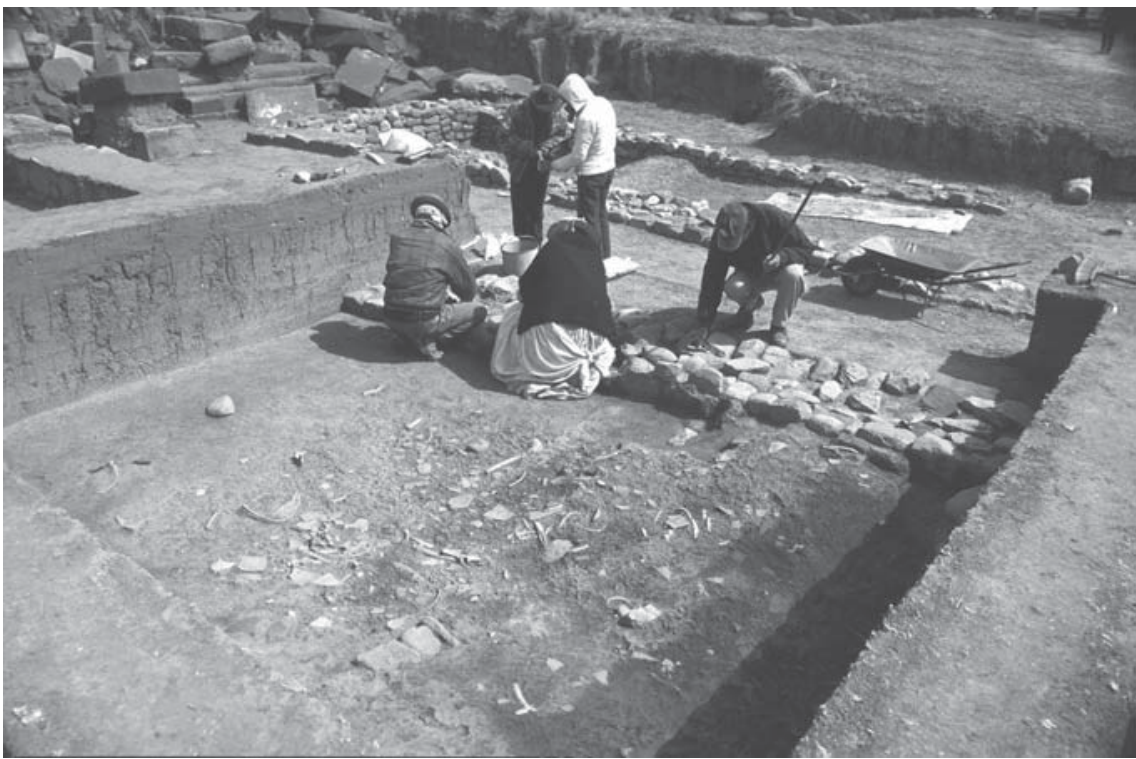

Figura 7: Depósito de desechos con mucho carbón, hueso de animales y fragmentos de ollas y aríbalos. Refuse deposit rich in charcoal, faunal material, olla and aribalous sherds.

dos quizás en banquetes festivos o actividades asociadas a éstos, lo cual indica un abundante consumo de carne y chicha.
Al este de las mencionadas estructuras se halla una plaza amplia, probablemente la zona de consumo de los alimentos mencionados, y la puer- 
ta que daba acceso del recinto donde se preparaba la comida a la plaza se encuentra demarcada por una puerta de doble jamba, revelando la entrada a un espacio de importancia política y ritual.

Finalmente, se evidencia la existencia de una serie de cámaras pequeñas construidas en las terrazas de Pumapunku. Su función queda aún sin conocer, por la carencia de artefactos in situ. Sin embargo, vale señalar que las cámaras muestran características arquitectónicas particulares, sobre todo en la amplitud que presentan los ingresos, que sugieren actividades expuestas a la vista del público, quizás con la intención de exponer determinados objetos o actividades al público que se encontraba en la plaza al este de las estructuras cuadrangulares. Debe argüirse en este punto que a pesar de que el espacio interior de las cámaras es menor a $2 \mathrm{~m}$, los ingresos a éstas llegan casi a los 2 $\mathrm{m}$ y su orientación hacia el este los hace particularmente visibles en la plaza, además se hallan asociados grandes bloques monolíticos de andesita dispuestos entre la plaza y la primera plataforma de la estructura.

\section{Conclusiones}

Para finalizar, volvemos a la pregunta que impulsó esta investigación, no sin antes hacer notar que las conclusiones son necesariamente preliminares, debido entre otras razones a la necesidad de contar con los resultados de los diversos análisis que aún se llevan a cabo.

A pesar de ello, ensayamos la hipótesis de que los Inkas escogieron a Pumapunku como el sector político-ceremonial de su asentamiento no por ser la estructura en mejor estado de conservación durante el Intermedio Tardío, sino porque, más que cualquier otra estructura, encajó con las demandas y requisitos de los ritos Inkas, como ya se mencionó con anterioridad.

La orientación de su eje que se relaciona con el pico del Illimani y que permitió que la pirámide fuese concebida como una imitación de dicho pico, dio lugar a la vez a una reinterpretación del paisaje existente y le otorgó una significancia particular que interactuaba con las características de planta y arquitectura presentes en la estructura. Esto pudo servir de base para su interpretación como un axis mundi en el que se combinaban los distintos planos de la concepción cosmológica aymara y quechua. A esta concepción se suman la presencia de la plaza que fue adecuada para las ceremonias Inkas y la escultura antropomorfa que probaba el mito que sugiere la actividad creadora de Viracocha en el sitio.

Los Inkas modificaron Pumapunku para que el espacio sagrado fuera más apropiado para sus ritos y correspondiera mejor a su cosmología, identificándolo quizás con un ushnu que servía de nexo entre los distintos planos cosmológicos existentes. Además dedicaron y santificaron de nuevo a la pirámide con ofrendas, asociadas a ricos ajuares que incluían pequeñas vasijas con las formas más emblemáticas del imperio. Por otra parte, construyeron un baño ceremonial y un sistema para transportar el agua desde el centro $\mathrm{Pu}$ mapunku al tanque ubicado al oeste. Hechos que sin duda muestran el importante rol de la estructura para las ceremonias Inkas.

Con lo expuesto hasta el momento, podemos ver la historia-mítica de la Creación "escrita" en las piedras del Pumapunku, dado que evidentemente algunos aspectos de la estructura hacían referencia a la creación. Por ejemplo, el agua podría señalar el lago Titicaca, lugar de origen de Viracocha, y el monolito que se hallaba en la zona pudo haber sido interpretado como un modelo usado por la deidad en la creación de las primeras personas.

Finalmente, ¿qué hacían los Inkas en los espacios remodelados alrededor de Pumapunku para invocar y materializar esta historia? La plaza pública para ceremonias y exhibiciones, con estructuras adyacentes para la preparación de banquetes festivos, sugiere un papel importante para las ceremonias. Y las áreas dedicadas a los banquetes y festividades al igual que la estructura formal permitían la reproducción y consolidación no sólo de los ritos, sino de la concepción misma de la estructura que hacía de ésta un lugar sagrado y de contacto con otros espacios espirituales.

Agradecimientos: Reconocemos a las personas e instituciones que han posibilitado nuestras investigaciones en Tiwanaku. Agradecemos a todo el personal de la Dirección Nacional de Arqueología (DINAR) por el apoyo que nos han brindado, en especial al Director Lic. Javier Escalante, al jefe de Conservación Eduardo Pareja y Freddy Arce. También reconocemos la ayuda del Director del Museo Nacional de Arqueología, Lic. César Velázquez. Agradecemos de igual manera la ayuda proporcionada por la Alcaldía y la Junta de Vecinos de Tiahuanaco y los Mallkus de la Central Agraria que representa a las comunidades de Tia- 
huanaco. Hacemos extensiva nuestra deuda a la Asociación de Trabajadores en Arqueología de Tiwanaku (A.S.T.A.T), la cual proporcionó el personal necesario para las labores de excavación y conservación. También agradecemos al equipo profesional del proyecto, y en especial al Dr. Alexei Vranich, director del Proyecto Arqueológico Pumapunku-Akapana, y al arqueólogo Marcelo Maldonado Vargas. Reconocemos la ayuda económica de la National Geographic Society, la H. John Heinz
III Fund for Latin American Archaeology y la Graduate School y la Vilas Foundation de la Universidad de Wisconsin. Finalmente, queremos reconocerles a Paola González y Mauricio Uribe Rodríguez por sus labores de organización, por reunir y editar las contribuciones en este número de Chungara, y también agradecer los comentarios y observaciones de dos evaluaciones anónimas, consideración de los cuales nos hizo aclarar y reforzar algunos aspectos de nuestro argumento.

\section{Referencias Citadas}

Albarracín-Jordan, J.

1996 Tiwanaku: Arqueología Regional y Dinámica Segmentaria. Editores Plural, La Paz.

1999 Arqueología de Tiwanaku. Historia de una Antigua Civilización Andina. SIGLA S.R.L., La Paz.

Albornoz, C. de

1989 [1581-1585] Instrucción para descubrir todas las guacas del Piru y sus camayos y haziendas. En Fábulas y Mitos de los Inkas, editado por H. Urbano y P. Duviols, pp. 163-198. Historia 16, Madrid.

Alcock, S.E.

2001 The reconfiguration of memory in the eastern Roman Empire. En Empires, editado por S.E. Alcock, T.N. D’Altroy, K.D. Morrison y C.M. Sinopoli, pp. 323-350. Cambridge University Press, Cambridge.

Arellano López, J.

1991 The new cultural contexts of Tiahuanaco. En Huari Administrative Structure, editado por W.H. Isbell y G.F. McEwan, pp. 259-280. Dumbarton Oaks, Washington, DC.

Basso, K.H.

1996 Wisdom sits in places: Notes on a western Apache landscape. En Senses of Place, editado por S. Feld y K.H. Basso, pp. 53-90. School of American Research Press, Santa Fe.

Bauer, B.S.

1998 The Sacred Landscape of the Inka: The Cusco Ceque System. University of Texas Press, Austin.

Bauer, B.S. y C. Stanish

2001 Ritual and Pilgrimage in the Ancient Andes: The Islands of the Sun and the Moon. University of Texas Press, Austin.

Berenguer, J. y P. Dauelsberg

1989 El norte grande en la órbita de Tiwanaku. En Culturas de Chile, Prehistoria, desde sus Orígenes hasta los Albores de la Conquista, editado por J. Hidalgo L., V. Schiappacasse F., H. Niemeyer F., C. Aldunate del S., e I. Solimano R., pp. 129-180. Editorial Andrés Bello, Santiago.

Betanzos, J. de

1987 [1551-1557] Suma y Narración de los Inkas. Ediciones Atlas, Madrid.

Bradley, R.

1998 The Significance of Monuments: On the Shaping of Human Experience in Neolithic and Bronze Age Europe. Routledge Press, London.
2000 An Archaeology of Natural Places. Routledge Press, London.

Binford, M.W., A.L. Kolata, M. Brenner, J.W. Janusek, M.T. Seddon, M. Abbott y J.H. Curtis

1997 Climate variation and the rise and fall of an Andean civilization. Quaternary Research 47(2):235-248.

Browman, D.L.

1978 Toward the development of the Tiahuanaco (Tiwanaku) State. En Advances in Andean Archaeology, editado por D.L. Browman, pp. 327-349. Mouton, Hague.

1981 New light on Andean Tiwanaku. American Scientist 69(4):408-419.

1997 Political institutional factors contributing to the integration of the Tiwanaku state. En Emergence and Change in Early Urban Societies, editado por L. Manzanilla, pp. 229-244. Plenum, New York.

Burger, R.L.

1992 Chavin and the Origins of Andean Civilization. Thames and Hudson, New York.

Cieza de León, P.

1959 [1553] Crónica del Perú, Primera y Segunda Parte. Fondo Editorial, Pontificia Universidad Católica del Perú, Lima.

Cobo, B. de

1939 Del templo y edificios de Tihuanacu. En Tihuanacu: Antología de los Principales Escritos de los Cronistas Coloniales, Americanistas e Historiadores Bolivianos, editado por G. A. Otero, pp. 28-44. Ministerio de Educación, Bellas Artes y Asuntos Indígenas, La Paz, Bolivia.

1990 Inka Religion and Customs. University of Texas Press, Austin.

Cook, A.G.

1994 Wari y Tiwanaku: Entre el Estilo y la Imagen. Fondo Editorial, Pontificia Universidad Católica del Perú, Lima.

Cordero Miranda, G.

1978 Informe Preliminar acerca de las excavaciones en Pumapunku. Informe al INAR, no. 33/78.

Cummins, T.B.F.

2002 Toasts with the Inka: Andean Abstraction and Colonial Images on Quero Vessels. University of Michigan Press, Ann Arbor.

Demarest, A.A.

1981 Viracocha: The Nature and Antiquity of the Andean High God. Peabody Museum Monograph No. 6. Peabody 
Museum of Archaeology and Ethnology, Harvard University, Cambridge.

Erickson, C

1999 Neo-environmental determinism and agrarian "collapse" in Andean prehistory. Antiquity 73:634-642.

Escalante Moscoso, J.

1997 Arquitectura Prehispánica en los Andes Bolivianos. Tercera edición, Producciones CIMA, La Paz.

Grant, F.J.

1893 Impressions of Tiahuanacu. La Paz-Bolivia.

Gutiérrez de Santa Clara, P.

1904 [1603] Historia de las Guerras Civiles del Perú(15441548) y de otros Sucesos de las Indias. Colección de los libros y documentos referentes a la historia de América, Madrid.

Janusek, J.W.

1994 State and Local Power in a Prehispanic Polity: Changing Patterns of Urban Residence in Tiwanaku and Lukurmata. Doctoral Dissertation, Department of Anthropology, University of Chicago, Chicago.

1999 Craft and local power: Embedded specialization in Tiwanaku cities. Latin American Antiquity 10(2):107-131. 2004 Household and City in Tiwanaku. En Andean Archaeology, editado por Helaine Silverman. London, Blackwell.

Kolata, A.L.

1982 Tiwanaku: Portrait of an Andean civilization. Field Museum of Natural History Bulletin 53:13-28.

1993 The Tiwanaku: Portrait of an Andean Civilization. Blackwell, Oxford.

Kolata, A.L., editor

1996 Tiwanaku and its Hinterland: Archaeology and Paleoecology of an Andean Civilization. Vol. 1: Agroecology. Smithsonian Institution Press, Washington, DC.

2003 Tiwanaku and its Hinterland: Archaeology and Paleoecology of an Andean Civilization. Vol. 1: Urban and Rural Archaeology. Smithsonian Institution Press, Washington, DC

Kolata, A.L. y C. Ponce Sanginés

1992 Tiwanaku: The city at the center. En The Ancient Americas: Art from Sacred Landscapes, editado por R. Townsend, pp. 317-335. Art Institute of Chicago, Chicago.

Manzanilla, L.

1992 Akapana. Una Pirámide en el Centro del Mundo. Instituto de Investigaciones Antropológicas, Universidad Nacional Autónoma de México, México, DF.

Moore, J.D.

1996 The archaeology of plazas and the proxemics of ritual: Three Andean traditions. American Anthropologist 98(4)7:789-802.

Ortloff, C. y A. Kolata

1993 Climate and collapse: Agro-ecological perspectives on the decline of the Tiwanaku State. Journal of Archaeological Science 20:195-221.

Parsons, J.R.

1968 An estimate of size and population for Middle Horizon Tiahuanaco. American Antiquity 33:243-245.

Ponce Sanginés, C.

1972 Tiwanaku: Espacio, Tiempo y Cultura: Ensayo de Síntesis Arqueológica. Primera edición. Academia Nacional de Ciencias de Bolivia. Publicación 30. La Paz.
1981 Descripción sumaria del Templete Semisubterráneo de Tiwanaku. Editorial Librería Juventud, La Paz.

1999 Tiwanaku: 200 años de Investigaciones Arqueológicas, Segunda edición. Producciones CIMA, La Paz.

Posnansky, A.

1945 Tihuanacu: The Cradle of American Man, Vols. I y II. J.J. Augustin, New York.

Protzen, J.P. y S.E. Nair

2001 Pumapunku: plataformas y portales. En Huari y Tiwanaku: Modelos vs. Evidencias (Segunda parte), pp. 309336. Boletín de Arqueología 5. Fondo Editorial de la Pontificia Universidad Católica del Perú, Lima.

2002 The gateways of Tiwanaku: Symbols or passages? En Andean Archaeology II: Art, Landscape, and Society, editado por H. Silverman y W.H. Isbell, pp. 189-223. Kluwer Academic/Plenum Publishers, New York.

Sarmiento de Gamboa, P.

1967 [1572] History of the Inkas. Kraus Reprint, Millwood. Sherbondy, J.

1992 Water ideology in Inka ethnogenesis. En Andean Cosmologies through Time: Persistence and Emergence, editado por R.V.H. Dover, K. Seibold, and J.H. McDowell, pp. 46-66. Indiana University Press, Bloomington.

Smith, A.E.

2002 Imperial Ideology and the Inka Occupation of Tiwa$n a k u$. Tesis de licenciatura, Northwestern University, Evanston, Illinois.

Stanish, C.

2002 Tiwanaku political economy. En Andean Archaeology I: Variations in Sociopolitical Organization, editado por W.H. Isbell y H. Silverman, pp. 169-198. Kluwer Academic / Plenum Publishers, New York.

Topic, J.R., T.L. Topic y A. Melly Cava

2002 Catequil: The archaeology, ethnohistory, and ethnography of a major provincial huaca. En Andean Archaeology I: Variations in Sociopolitical Organization, editado por W.H. Isbell y H. Silverman, pp. 303-336. Kluwer Academic / Plenum Publishers, New York.

Rostworowski de Diez Canseco, M.

1992 Pachacamac y el Señor de los Milagros: Una Trayectoria Milenaria. Instituto de Estudios Peruanos, Lima.

Vranich, A.

1999 Interpreting the Meaning of Ritual Spaces: the Temple Complex of Pumapunku, Tiwanaku, Bolivia. Tesis doctoral, University of Pennsylvania. Philadelphia.

2001 La pirámide de Akapana: reconsiderando el centro monumental de Tiwanaku. En Huari y Tiwanaku: Modelos vs. Evidencias (Segunda parte), pp. 295-308. Boletín de Arqueología 5. Fondo Editorial de la Pontificia Universidad Católica del Perú, Lima.

Vranich, A. y J. Yaeger

2003 The social life of a monument: Tiwanaku, the Pumapunku, and the origin of the Inka ushnu. Ponencia presentada en la 102 reunión de la American Anthropological Association, Chicago.

Vranich, A., J.M. López B., J. Yaeger y M. Maldonado Vargas 2002 Excavaciones Arqueológicas del Proyecto Arqueológico Pumapunku-Akapana en Tiwanaku, Gestión 2001. Informe depositado en DINAR, La Paz, Bolivia. 
Vranich, A., J. Yaeger, M. Bruno y J. Burkholder 1999 Digital Photography and Archaeological Methodology: The 1999 Tiwanaku Project. Informe depositado con DINAR, La Paz, Bolivia.

Vranich, A., J. Yaeger, D. Villamor, H.R. Haines, J.M. López Bejarano, M. Maldonado Vargas y E. Tabas.
2001 Excavaciones Arqueológicas en la Pirámide de Pumapunku, Tiwanaku. Informe depositado en DINAR, La Paz, Bolivia.

Zuidema, R.T.

1990 Inka Civilization in Cuzco. University of Texas Press, Austin. 